\title{
The Truncated Power Lindley distribution: Model, Properties and Applications
}

\author{
Mahmoud.A.Eltehiwy \\ Faculty of the Politics and Economics, \\ Department of Statistics, \\ Beni-suef University, Egypt. \\ Email: Mahmoud.abdelmone@eps.bsu.edu.com
}




\title{
The Truncated Power Lindley distribution: Model, Properties and Applications
}

\begin{abstract}
In this paper, we propose the truncated version of the power Lindley distribution which is more flexible than many well-known distributions. Mathematical and statistical properties of truncated power Lindley distribution are given only for upper truncated version of power Lindley distribution. The statistical proprieties such as moments, quantile function and order statistics are also discussed. The maximum likelihood estimators are constructed for estimating the unknown parameters of the upper, lower and double truncated power Lindley distributions. A set of real data containing the strengths of the glass of aircraft window, is considered to show the applicability of the truncated power Lindley distributions. The real data set is considered to illustrate the utility and potential of the proposed model.
\end{abstract}

Keywords and Phrases: Truncated power Lindley distribution, Moments, Quantile function, Order statistics, Maximum likelihood estimator.

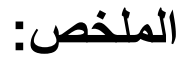

في هذه البحث، نقترح نوزيع جديد يسمى the truncated power Lindley. قام الباحث باشتقاق العديد

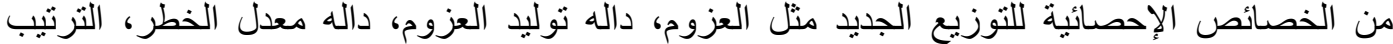

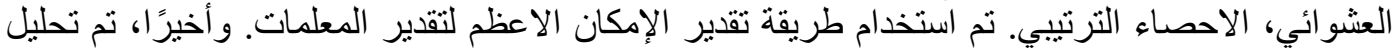

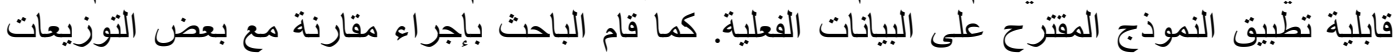
الموجودة.

Corresponding author

Name: Mahmoud Eltehiwy

Affiliation: faculty of the politics and Economics, Department of Statistics, BeniSuef University, Egypt

Tel: 01129379756

E-mail address: Mahmoud.abdelmone@eps.bsu.edu.eg

$$
\begin{aligned}
& \text { الاسم: محمود عبد المنعم محمد التحيوى التئي } \\
& \text { عنوان العمل : كليه السياسه و الاقتصاد - قسم الإحصاء- جامعه بنى سويف } \\
& \text { تليفون: } 01129379756 \\
& \text { الايميل الجامعى: Mahmoud.abdelmone@eps.bsu.edu.eg }
\end{aligned}
$$




\section{Introduction}

A truncated distribution is defined as a conditional distribution that results from restricting the domain of the statistical distribution. Hence, truncated distributions are used in cases where occurrences are limited to values which lie above or below a given threshold or within a specified range. If occurrences are limited to values which lie below a given threshold, the lower (left) truncated distribution is obtained. Similarly, if occurrences are limited to values which lie above a given threshold, the upper (right) truncated distribution arises. Truncated versions of the well-known statistical distributions are proposed by many researchers to model the truncated data in various fields. Zhang and Xie (2011) studied the characteristics of the truncated Weibull distribution and illustrated the applicability of this distribution to modeling lifetime data. Ahmed et al. (2010) proposed the truncated version of the Birnbaum-Saunders (BS) distribution and showed that truncated BS distribution is more appropriate than the classical BS model for describing the financial loss data from a commercial bank. Recently, Singh et al. (2014) have introduced the truncated version of the Lindley distribution and discussed statistical properties of proposed distribution and showed that truncated version of the Lindley distribution provides a better modeling than Weibull, Lindley and exponential distributions based on a real data.

From the above commentary and monitoring the wide applicability of the truncated distributions, we proposed the truncation in the power Lindley distribution. Lindley (1958), introduced a one-parameter distribution, known as Lindley distribution, given by its probability density function 


$$
\mathrm{g}(t ; \theta)=\frac{\theta^{2}}{1+\theta}(1+t) e^{-\theta t} ; \quad t>0, \theta>0
$$

It can be seen that this distribution is a mixture of exponential $(\theta)$ and gamma $(2, \theta)$ distributions. Its cumulative distribution function has been obtained as

$$
G(t)=1-\frac{\theta+1+\theta t}{\theta+1} e^{-\theta t} \quad ; t>0, \theta>0
$$

Since last decade, Lindley distribution has been attracting the attention of the researchers, scientists and the reliability probationers, and many author extended it to the various distributions. Ghitany et al. (2008) have discussed various properties of this distribution and showed that in many ways that the pdf given by (1) provides a better model for some applications than the exponential distribution. Bakouch et al. (2012) obtained an extended Lindley distribution and discussed its various properties and applications. Ghitany et al. (2011) developed a twoparameter weighted Lindley distribution and discussed its applications to survival data. Nadarajah et al. (2011) obtained a generalized Lindley distribution and discussed its various properties and applications. Merovci and Elbatal (2014) use the quadratic rank transmutation map in order to generate a flexible family of probability distributions taking Lindley-geometric distribution as the base value distribution by introducing a new parameter that would offer more distributional flexibility and called it transmuted Lindley-geometric distribution. Asgharzadeh et al. (2014) introduced a general family of continuous lifetime distributions by compounding any continuous distribution and the Poisson-Lindley distribution. Oluyede and Yang (2015) proposed a new four-parameter class of generalized Lindley (GLD) distribution called the beta-generalized Lindley (BGLD) distribution. This class of distributions contains the beta-Lindley, GLD and Lindley distributions as special cases. Ashour and Eltehiwy (2015) obtained the 
exponentiated power Lindley distribution and discussed its various properties and applications

A two parameter power Lindley distribution (PLD), of which the Lindley distribution 'equation (1)' is a particular case, has been suggested by Ghitany et al. (2013). They introduced a new extension of the Lindley distribution by considering the power transformation of the r.v. $\mathrm{Y}=T^{1 / \beta}$. The pdf of the $Y$ is readily obtained to be Power Lindley distribution with parameters $\beta$ and $\theta$ and is defined by its probability density function pdf

$$
\mathrm{g}(y ; \theta)=\frac{\theta^{2} \beta\left(1+y^{\beta}\right)}{\theta+1} y^{\beta-1} e^{-\theta y^{\beta}} ; \quad y>0, \theta, \beta>0
$$

It can easily be seen that at $\beta=1$, the Equation (3) reduces to the Lindley distribution. From Equation (2), we see that the power Lindley distribution is a two-component mixture of Weibull distribution (with shape $\beta$ and scale $\theta$ ), and a generalized gamma distribution (with shape parameters $2 \beta$ and scale $\theta$ ), with mixing proportion $p=\frac{\theta}{1+\theta}$.

$$
\mathrm{g}(y ; \beta ; \theta)=p f_{1}(y)+(1-p) f_{2}(y)
$$

where

$$
\begin{gathered}
p=\frac{\theta}{1+\theta^{\prime}} \\
f_{1}(y)=\theta \beta y^{\beta-1} e^{-\theta y^{\beta}}, y>0, \theta, \beta>0
\end{gathered}
$$

and

$$
f_{2}(y)=\theta^{2} \beta y^{2 \beta-1} e^{-\theta y^{\beta}}, y>0, \theta, \beta>0
$$


Some extensions of the Lindley distributions e.g. power Lindley and generalized Lindley distributions etc. are the good competitors of the Weibull distribution and can be quite effectively used to model the real phenomenon where the Weibull distribution seems to be incompatible to the real data. In this direction, one can also study the properties of truncated versions of these Lindley's generalizations as the alternative models to the truncated Weibull distribution in the literature. Therefore, this article aims to start the discussions with introducing the concept of the truncation in two parameter power Lindley distribution.

The rest of the paper is arranged in the following sections. In section 2, the truncated versions of the power Lindley distribution, named as the upper truncated power Lindley (UTPL), lower truncated power Lindley (LTPL), double truncated power Lindley (DTPL) distributions are introduced. Particularly, the flexibility of the UTPL distribution has been shown demonstrating the characteristics of the probability density (pdf) and hazard functions with different combination of the values of its parameters. The moments, moment generating function, quantile function, skewness and kurtosis and order statistics of the UTPL distribution are derived in section 3 . In section 4 , the method of the maximum likelihood is applied to obtain the estimates of the parameters of the UTPL, LTPL and DTPL distributions. In section 5, a set of real data is modeled through the different distributions and their applicability is compared. Finally, the paper is concluded in section 6 .

\section{The truncated power Lindley distributions}

A distribution $G(x ; \Theta)$ is said to be a double truncated distribution over the interval $[v, \zeta]$ if it has the cumulative distribution function (cdf) defined as 


$$
G(x ; \Theta)=\frac{F(x ; \Theta)-F(v ; \Theta)}{F(\zeta ; \Theta)-F(v ; \Theta)}, \quad v \leq x \leq \zeta \quad-\infty<v<\zeta<\infty
$$

and probability density function (pdf) is

$\mathrm{g}(x ; \Theta)=\frac{f(x ; \Theta)}{F(\zeta ; \Theta)-F(v ; \Theta)}, v \leq x \leq \zeta \quad-\infty<v<\zeta<\infty$

where, $f(x ; \Theta)$ and $F(x ; \Theta)$ are the pdf and cdf of the baseline model and $\Theta \in \mathcal{R}^{\mathrm{n}}$ denotes the vector parameter of base line model. Here, three cases can be recognized as

I. When $v=0$ and $\zeta \rightarrow \infty$, it reduces to baseline model.

II. When $v=0$, it is called the upper truncated distribution of the baseline model.

III. When $\zeta \rightarrow \infty$, it is called the lower truncated distribution of the baseline model.

In this article, we consider the power Lindley distribution as baseline model with the following distribution function :

$$
F(x)=\left[1-\left(1+\frac{\theta x^{\beta}}{\theta+1}\right) e^{-\theta x^{\beta}}\right]
$$

Using (7) and (9), the double truncated power Lindley distribution is defined as

$$
\mathrm{g}_{D}(x ; \theta, \beta)=\frac{\theta^{2}}{\theta+1} \frac{\beta\left(1+x^{\beta}\right) x^{\beta-1} e^{-\theta x^{\beta}}}{F(\zeta ; \Theta)-F(v ; \Theta)} \quad, \quad 0 \leq v \leq x \leq \zeta<\infty
$$

In the following sections, we will only discuss the properties of the upper truncated power Lindley distribution and the same procedure can be applied to study the properties of the lower truncated power Lindley distribution as well as double truncated power Lindley distribution. The upper truncated power Lindley distribution has the following pdf is given by 


$$
\mathrm{g}_{u}(x ; \theta, \beta)=\frac{\theta^{2} \beta\left(1+x^{\beta}\right) x^{\beta-1} \exp \left(-\theta\left(x^{\beta}-\zeta^{\beta}\right)\right)}{(\theta+1)\left(\exp \left(\theta \zeta^{\beta}\right)-1\right)-\theta \zeta^{\beta}} ; \quad 0 \leq x \leq \zeta
$$

It is denoted by $\operatorname{UTPL}(\theta, \beta, \zeta)$. Note that the above pdf will behave like as

i. $\quad \frac{d}{d x} \mathrm{~g}(x ; \theta ; \beta)=\mathrm{g}^{\prime}(x)=\frac{\theta^{2} \beta x^{\beta-2} \exp \left(-\theta\left(x^{\beta}-\zeta^{\beta}\right)\left[-\beta \theta x^{2 \beta}+(2 \beta-1-\theta \beta) x^{\beta}+\beta-1\right]\right.}{(\theta+1)\left(\exp \left(\theta \zeta^{\beta}\right)-1\right)-\theta \zeta^{\beta}}$

ii. Decreasing if
a) $\left\{0<\beta \leq \frac{1}{2}, \theta>0\right\}$;
b) $\left\{\frac{1}{2}<\beta<1, \theta \geq \eta_{1}(\beta)\right\}$, where $\eta_{1}(\beta)=\frac{1-2 \sqrt{\beta(1-\beta)}}{\beta}$,
c) $\{\beta=1, \theta \geq 1\}$;

iii. Unimodal if
a) $\{\beta=1, \theta>0$,$\} ; and mode values is x_{M_{0}}=\frac{(1-\theta)}{\theta}$
b) $\{\beta>1, \theta>0\}$.

iv. Decreasing- increasing- decreasing if $\left(\frac{1}{2}<\beta<1,0<\theta<\eta_{1}(\beta)\right)$

Figure 1 illustrates some of the possible shapes of the density function of truncated power Lindley distribution for selected values of the parameters $(\theta, \beta, \zeta)$.

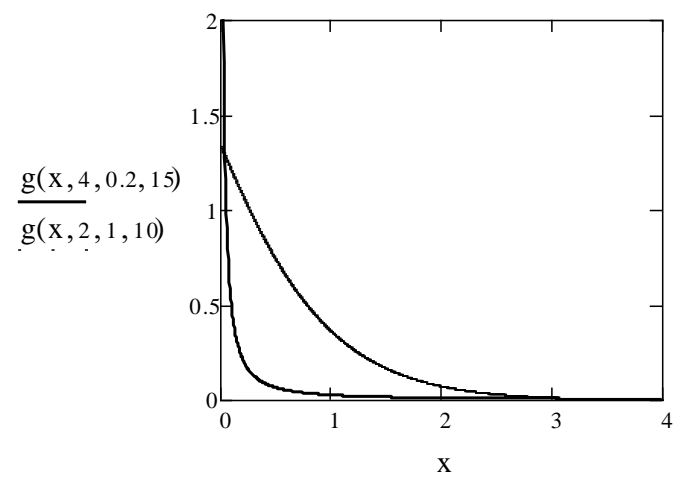

(a) 


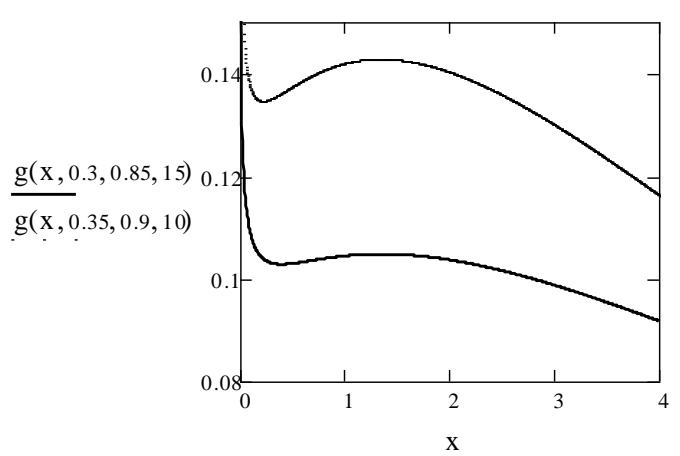

(c)

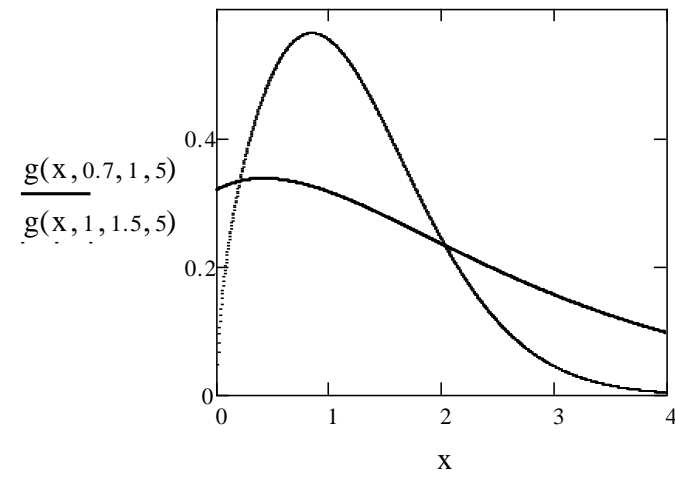

(b)

Figure 1: Probability density function of UTPL distribution for the cases

a) $(\theta, \beta, \zeta):(4,0.2,15)$ (solid), $(2,1,10)$ (dashed),

b) $(\theta, \beta, \zeta):(0.7,1.5,5)$ (solid), $(1,1.5,5)$ (dashed),

c) $(\theta, \beta, \zeta):(0.3,0.85,15)$ (solid), $(0.35,0.9,10)$ (dashed).

The corresponding hazard function at epoch $t$ is given by

$$
h(t ; \theta)=\frac{\theta^{2}}{\theta+1} \frac{\beta\left(1+t^{\beta}\right) t^{\beta-1} e^{-\theta t} t^{\beta}}{F(\zeta ; \Theta)-F(t ; \Theta)} ; \quad 0 \leq t \leq \zeta
$$

The behavior of $h(x)$ at $t=0$ and $t=\infty$, respectively, are given by

$$
h(0)=\left\{\begin{array}{cc}
\infty, & \text { if } \beta<1, \\
\frac{\theta^{2}}{\left[(\theta+1)\left(1-e^{-\theta \zeta}\right)-\theta \zeta e^{-\theta \zeta}\right]}, & \text { if } \beta=1, \\
0, & \text { if } \beta>1,
\end{array}\right.
$$

$h(\infty)= \begin{cases}0, & \text { if } \beta<1 \\ 0, & \text { if } \beta=1 \\ \infty, & \text { if } \beta>1\end{cases}$

$h(\zeta)=\infty$, i.e. as $t \rightarrow \zeta, h(t) \rightarrow \infty$

Figure 2 shows the HRF $h(x)$ of the truncated power Lindley distribution for some choices of values of the parameters $(\theta, \beta, \zeta)$. 


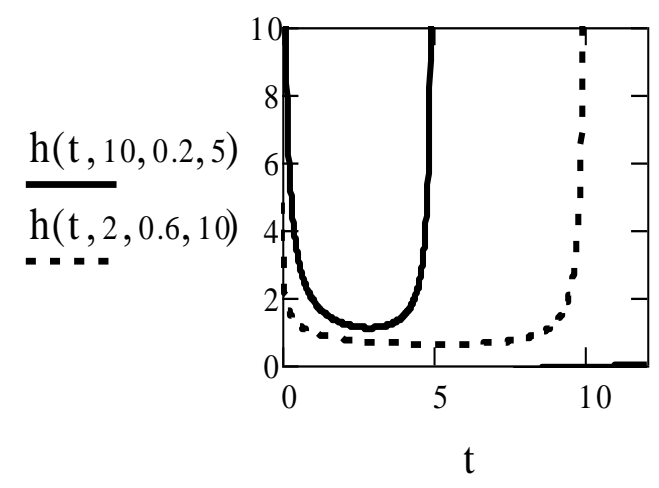

(a)

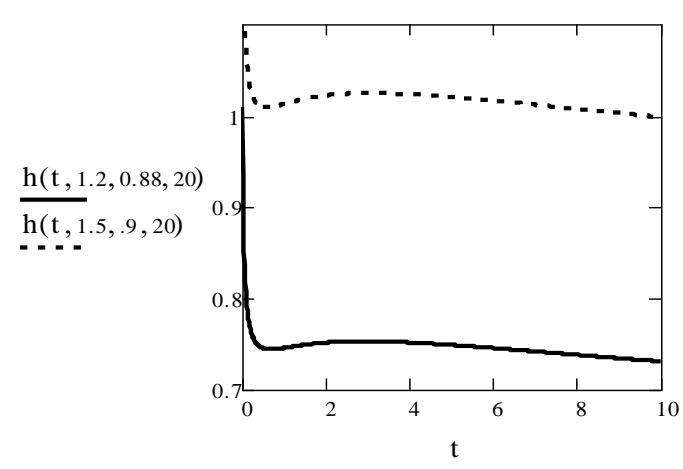

(c)

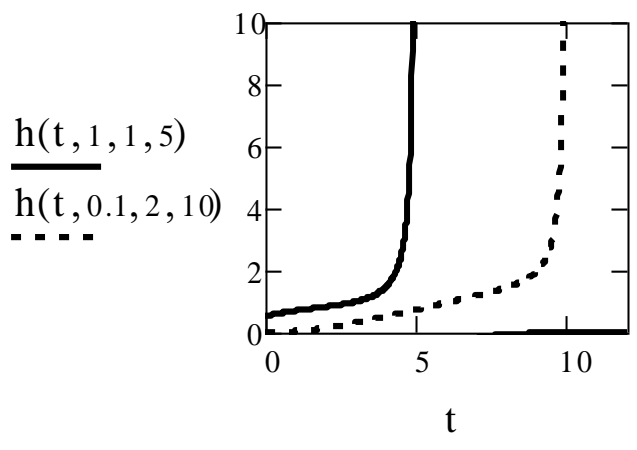

(b)

Figure 2: hazard rate function of UTPL distribution for the cases

a) $(\theta, \beta, \zeta):(10,0.2,5)$ (solid), $(2,0.6,10)$ (dashed), b) $(\theta, \beta, \zeta):(1,1,5)$ (solid), $(0.1,2,10)$ (dashed), c) $(\theta, \beta, \zeta):(1.2,0.88,20),($ solid $),(1.5,0.9,20)$ (dashed).

\section{Statistical properties}

\subsection{Moments and related measures}

The $r$ th raw moment under the upper truncated power Lindley distribution is defined as 


$$
\begin{aligned}
& E\left[X^{r}\right]=\frac{\theta^{2} \beta}{(1+\theta)} \frac{1}{F(\zeta ; \Theta)} \int_{0}^{z e t a} x^{r+\beta-1}\left(1+x^{\beta}\right) e^{-\theta x^{\beta}} d x \\
& =\frac{\theta^{2} \beta}{(1+\theta)} \frac{1}{F(\zeta ; \Theta)} \int_{0}^{\text {zeta }} x^{r+\beta-1} e^{-\theta x^{\beta}} d x+\frac{\theta^{2} \beta}{(1+\theta)} \frac{1}{F(\zeta ; \Theta)} \int_{0}^{\text {zeta }} x^{r+2 \beta-1} e^{-\theta x^{\beta}} d x
\end{aligned}
$$

We make the following bijective continuously differentiable variable change:

Let $u=\theta x^{\beta} \quad, \quad x=\left(\frac{u}{\theta}\right)^{\frac{1}{\beta}}, \quad d x=\frac{\left(\frac{u}{\theta}\right)^{\frac{1}{\beta}-1}}{\beta \theta} d u$

We then get :

$$
\begin{aligned}
\mu_{r}^{\prime} & =E\left[X^{r}\right]=\frac{\theta}{(1+\theta) \theta^{\frac{r}{\beta}} F(\zeta ; \Theta)} \int_{0}^{\theta \zeta^{\beta}} u^{\frac{r}{\beta}} e^{-u} d u+\frac{\theta}{(1+\theta) \theta^{\frac{r}{\beta}+1} F(\zeta ; \Theta)} \int_{0}^{\theta \zeta^{\beta}} u^{\frac{r}{\beta}+1} e^{-u} d u \\
& =\frac{\theta}{(1+\theta) \theta^{\frac{r}{\beta}} F(\zeta ; \Theta)} \gamma\left(\frac{r}{\beta}+1, \theta \zeta^{\beta}\right)+\frac{1}{(1+\theta) \theta^{\frac{r}{\beta}} F(\zeta ; \Theta)} \gamma\left(\frac{r}{\beta}+2, \theta \zeta^{\beta}\right) \\
& =\frac{1}{(1+\theta) \theta^{\frac{r}{\beta}} F(\zeta ; \Theta)}\left[\theta \gamma\left(\frac{r}{\beta}+1, \theta \zeta^{\beta}\right)+\gamma\left(\frac{r}{\beta}+2, \theta \zeta^{\beta}\right)\right],
\end{aligned}
$$

where $\gamma$ denotes the incomplete Gamma function defined as :

$\forall z \in \mathbb{R}_{*}^{+}, \forall u \in \mathbb{R}^{+}, \quad \gamma(z, u)=\int_{0}^{u} x^{z-1} e^{-x} d x$

The mean and variance of UTPL distribution can be written as:

$$
\begin{aligned}
& \mu=E(X)=\frac{\left[\theta \gamma\left(\frac{1}{\beta}+1, \theta \zeta^{\beta}\right)+\gamma\left(\frac{1}{\beta}+2, \theta \zeta^{\beta}\right)\right]}{(1+\theta) \theta^{\frac{r}{\beta}}\left(1-\left(1+\frac{\theta \zeta^{\beta}}{\theta+1}\right) e^{-\theta \zeta^{\beta}}\right)}, \\
& \sigma^{2}=V(X)=E(X-E(X))^{2}=\mu_{2}^{\prime}-\left[\mu_{1}^{\prime}\right]^{2}
\end{aligned}
$$




$$
=\frac{\left[\theta \gamma\left(\frac{2}{\beta}+1, \theta \zeta^{\beta}\right)+\gamma\left(\frac{2}{\beta}+2, \theta \zeta^{\beta}\right)\right]}{(1+\theta) \theta^{\frac{r}{\beta}}\left(1-\left(1+\frac{\theta \zeta^{\beta}}{\theta+1}\right) e^{-\theta \zeta^{\beta}}\right)}-\left(\frac{\left[\theta \gamma\left(\frac{1}{\beta}+1, \theta \zeta^{\beta}\right)+\gamma\left(\frac{1}{\beta}+2, \theta \zeta^{\beta}\right)\right]}{(1+\theta) \theta^{\frac{r}{\beta}}\left(1-\left(1+\frac{\theta \zeta^{\beta}}{\theta+1}\right) e^{-\theta \zeta^{\beta}}\right)}\right)^{2} .
$$

Table 1: Mean and variance of the UTPL distribution for several values of the parameters

\begin{tabular}{|c|c|c|c|c|c|c|c|c|c|}
\hline \multirow{2}{*}{$\theta$} & \multirow{2}{*}{$\beta$} & \multicolumn{2}{|c|}{$\zeta=5$} & \multicolumn{2}{|c|}{$\zeta=10$} & \multicolumn{2}{|c|}{$\zeta=15$} & \multicolumn{2}{|c|}{$\zeta=20$} \\
\hline & & $\mu$ & $\sigma^{2}$ & $\mu$ & $\sigma^{2}$ & $\mu$ & $\sigma^{2}$ & $\mu$ & $\sigma^{2}$ \\
\hline \multirow{4}{*}{0.} & 0.25 & .2325 & 2.025 & .5024 & .152 & .784 & 18.402 & .073 & 32.781 \\
\hline & 0.75 & .5483 & 2.115 & 5.1269 & 8.2 & 7.622 & 18.279 & 10.023 & 32.22 \\
\hline & 1 & 9174 & 1.8 & 5.7116 & 7.075 & 205 & 15. & 376 & 27.824 \\
\hline & 1.2 & 1657 & 1.55 & 5.8902 & 6.155 & 7.858 & 13.538 & 9.077 & 21.86 \\
\hline \multirow{4}{*}{0.25} & 25 & & .941 & 2.3174 & 7.762 & 3.478 & 17.44 & & 30.952 \\
\hline & 0.75 & .3471 & 2.133 & 4.4681 & 8.253 & 6.305 & 17.937 & 7.879 & 30.598 \\
\hline & 1 & .6373 & 1.899 & 4.6299 & 7.138 & 5.892 & 14.159 & 6.592 & 20.757 \\
\hline & $\overline{12}$ & 7 & 1 & & 5. & 578 & 7.8 & 26 & .445 \\
\hline \multirow{4}{*}{0.} & 25 & .0284 & 1.789 & 2.0194 & 7.04 & 2.986 & 15.638 & 3.933 & 27.506 \\
\hline & 0.75 & .0101 & 2.056 & 3.4007 & 7.233 & 4.298 & 13.745 & 4.854 & 20.093 \\
\hline & 1 & 1657 & 1.8 & 3.0634 & 5.133 & 3.287 & 6.92 & 3.327 & 7.433 \\
\hline & 1.25 & 1488 & 35 & 2.4784 & 2.707 & 2.486 & 2.773 & 2.487 & 2.774 \\
\hline \multirow{4}{*}{1} & 25 & 7953 & .455 & 1.48 & 5.445 & 2.108 & 11.668 & 2.697 & 19.932 \\
\hline & 0.75 & 3997 & 1.59 & 1.833 & 3.71 & 1.947 & 4.805 & 1.975 & 5.221 \\
\hline & 1 & 79 & 1.2 & 1.497 & 1.725 & 1.5 & 1.749 & 1.5 & 1.75 \\
\hline & & .2924 & 8 & 1.304 & 0.873 & 1.304 & 0.873 & 1.304 & 0.873 \\
\hline \multirow{4}{*}{1.5} & 0.25 & 5958 & 7 & 1.039 & 3.889 & 1.414 & 7.91 & 1.745 & 12.952 \\
\hline & 0.75 & 0.9505 & 1.022 & 1.053 & 1.571 & 1.062 & 1.667 & 1.063 & 1.68 \\
\hline & 1 & 0.9227 & 0.678 & 0.933 & 0.729 & 0.933 & 0.729 & 0.933 & 0.729 \\
\hline & 1.25 & 0.8885 & 0.434 & 0.889 & 0.435 & 0.889 & 0.435 & 0.889 & 0.435 \\
\hline
\end{tabular}

Table 1 shows that the mean and variance decrease when $\theta$ increase for a fixed $\beta$. besides, for a fixed $\theta$, while mean increase, variance decreases when $\beta$ increases. Assuming $\theta$ and $\beta$ is kept fixed, the mean and variance increase when $\zeta$ increases.

The skewness and kurtosis of the distribution can be simply verified by using the following relationship 


$$
\begin{gathered}
S_{k}=\frac{\left(\mu_{3}^{\prime}-3 \mu \mu_{2}^{\prime}+2 \mu^{3}\right)^{2}}{\left(\mu_{2}^{\prime}-\mu^{2}\right)^{3}} \\
K_{t}=\frac{\left(\mu_{4}^{\prime}-4 \mu \mu_{3}^{\prime}+6 \mu^{2} \mu_{2}^{\prime}-3 \mu^{4}\right)}{\left(\mu_{2}^{\prime}-\mu^{2}\right)^{2}}
\end{gathered}
$$

\subsection{Moment Generating Function}

Let $X$ have an upper truncated power Lindley distribution. Then the moment generating function of $X, M_{X}(t)$, is

$M_{X}(t)=M_{X}(t)=\frac{C_{j}}{(1+\theta)}\left[\frac{\Gamma\left(\frac{j}{\beta}+1\right)}{\theta^{\frac{j}{\beta}-1}}+\frac{\Gamma\left(\frac{j}{\beta}+2\right)}{\theta^{\frac{j}{\beta}}}\right]$,

where $C_{j}=\frac{1}{F(\zeta ; \Theta)} \sum_{j=0}^{\infty} \frac{t^{j}}{j !}$

Proof.

$$
\begin{gathered}
M_{X}(t)=\int_{0}^{\infty} e^{t x} \mathrm{~g}(x) d x \\
M_{X}(t)=\frac{\theta^{2} \beta}{(1+\theta)} \frac{1}{F(; ; \theta)} \int_{0}^{\infty} x^{\beta-1}\left(1+x^{\beta}\right) e^{t x} e^{-\theta x^{\beta}} d x
\end{gathered}
$$

Using the following expansion of $e^{t x}$ given by

$$
e^{t x}=\sum_{j=0}^{\infty} \frac{t^{j} x^{j}}{j !}
$$

Equation (13) can be rewritten as follow:

$$
\begin{aligned}
M_{X}(t)=\frac{\theta^{2} \beta}{(1+\theta)} \frac{1}{F(\zeta ; \Theta)} \sum_{j=0}^{\infty} \frac{t^{j}}{j !} \int_{0}^{\infty} x^{j+\beta-1} e^{-\theta x^{\beta}} d x \\
+\frac{\theta^{2} \beta}{(1+\theta)} \frac{1}{F(\zeta ; \Theta)} \sum_{j=0}^{\infty} \frac{t^{j}}{j !} \int_{0}^{\infty} x^{j+2 \beta-1} e^{-\theta x^{\beta}} d x
\end{aligned}
$$


Let $=x^{\beta}, d(t)=\beta x^{\beta-1} d x$ and $C_{j}=\frac{1}{F(\zeta ; \Theta)} \sum_{j=0}^{\infty} \frac{t^{j}}{j !}$

Equation (13) can be rewritten as follows:

$$
\begin{gathered}
M_{X}(t)=C_{j} \frac{\theta^{2}}{(1+\theta)} \int_{0}^{\infty} t^{\frac{j}{\beta}} e^{-\theta t} d t+C_{j} \frac{\theta^{2}}{(1+\theta)} \int_{0}^{\infty} x^{\frac{j}{\beta}+1} e^{-\theta t} d x \\
M_{X}(t)=\frac{C_{j}}{(1+\theta)} \frac{\Gamma\left(\frac{j}{\beta}+1\right)}{\theta^{\frac{j}{\beta}-1}}+\frac{C_{j}}{(1+\theta)} \frac{\Gamma\left(\frac{j}{\beta}+2\right)}{\theta^{\frac{j}{\beta}}}, \\
M_{X}(t)=\frac{C_{j}}{(1+\theta)}\left[\frac{\Gamma\left(\frac{j}{\beta}+1\right)}{\theta^{\frac{j}{\beta}-1}}+\frac{\Gamma\left(\frac{j}{\beta}+2\right)}{\theta^{\frac{j}{\beta}}}\right] .
\end{gathered}
$$

\subsection{Quantile Function}

Let $X$ denotes a random variable with the probability density function (Eq. 10). The quantile function, say $Q(p)$, defined by $F(Q(p))=p$ is the root of the equation

$$
\left[1+\frac{\theta[Q(p)]^{\beta}}{1+\theta}\right] \exp \left\{-\theta[Q(p)]^{\beta}\right\}=1-F(\zeta ; \Theta) p
$$

for $0<p<1$. Substituting $Z(p)=-1-\theta-\theta[Q(p)]^{\beta}$, one can rewrite equation (14) as

$$
Z(p) \exp \{Z(p)\}=\frac{-(1+\theta)(1-F(\zeta ; \Theta) p)}{\exp (1+\theta)}
$$

for $0<p<1$. So, the solution for $Z(p)$ is

$$
Z(p)=W\left(\frac{-(1+\theta)(1-F(\zeta ; \Theta) p)}{\exp (1+\theta)}\right)
$$


for $0<p<1$, where $W($.$) is the Lambert W$ function, see Corless et al. (1996) for detailed properties. Inverting (Eq. 15), one obtains

$$
Q(p)=\left[-1-\frac{1}{\theta}-\frac{1}{\theta} W\left(\frac{-(1+\theta)(1-F(\zeta ; \Theta) p)}{\exp (1+\theta)}\right)\right]^{1 / \beta}
$$

for $0<p<1$. As $\zeta \rightarrow \infty$, from the above equation (16), we get the quantile function of power Lindley distribution derived by Ghitani et al (2013). The particular case of (Eq. 16) for $(\zeta \rightarrow \infty, \beta=1)$ has been derived recently by Jodrá (2010).

The median of the UTPL distribution can be obtained as

$$
M d_{x}=\left[-1-\frac{1}{\theta}-\frac{1}{\theta} W\left(\frac{-(1+\theta)(2-F(\zeta ; \Theta))}{2 \exp (1+\theta)}\right)\right]^{1 / \beta}
$$

\subsection{Skewness and Kurtosis}

The effects of the parameters on the skewness and kurtosis of $\mathrm{X}$ can be based on quantile function in Eq. (16). There are many heavy tailed distributions for which this measure is infinite. So, it becomes uninformative precisely when it needs to be. The Bowley's skewness is based on quartiles:

$$
S=\frac{Q(3 / 4)-2 Q(1 / 2)+Q(1 / 4)}{Q(3 / 4)-Q(1 / 4)},
$$

and the Moors' kurtosis is based on octiles:

$$
\mathcal{M}=\frac{[Q(7 / 8)-Q(5 / 8)]+[Q(3 / 8)-Q(1 / 8)]}{Q(6 / 8)-Q(2 / 8)},
$$


where $Q($.$) represents the quantile function of \mathrm{X}$. These measures are less sensitive to outliers and they exist even for distributions without moments. Skewness measures the degree of the long tail and kurtosis is a measure of the degree of tail heaviness. When the distribution is symmetric, $S=0$ and when the distribution is right (or left) skewed, $>0$ (or $S<0)$. As $\mathcal{M}$ increases, the tail of the distribution becomes heavier.

For any distribution symmetrical to 0 the Moors kurtosis reduces to

$$
\mathcal{M}=\frac{(Q(7 / 8)-Q(5 / 8))}{Q(6 / 8)}
$$

It is easy to calculate that for standard normal distribution:

$Q(1 / 8)=-Q(7 / 8)=-1.15, Q(2 / 8)=-Q(6 / 8)=-0.32$. Therefore,

$\mathcal{M}=1.23$. Hence, the centered Moor's coefficient is given by

$$
\mathcal{M}=\frac{[Q(7 / 8)-Q(5 / 8)]+[Q(3 / 8)-Q(1 / 8)]}{Q(6 / 8)-Q(2 / 8)}-1.23
$$

From (Eq. 16), skewness and kurtosis of the UTPL distribution are obtained and presented in table 2 .

Table 2: Skewness and kurtosis of the UTPL distribution for several values of the parameters

\begin{tabular}{|c|c|l|l|l|l|l|l|}
\hline \multirow{2}{*}{$\theta$} & \multirow{2}{*}{$\beta$} & \multicolumn{2}{|c|}{$\zeta=5$} & \multicolumn{2}{c|}{$\zeta=10$} & \multicolumn{2}{c|}{$\zeta=15$} \\
\cline { 2 - 8 } & & Skewness & kurtosis & Skewness & kurtosis & Skewness & kurtosis \\
\hline \multirow{3}{*}{0.1} & 0.25 & -0.0987 & 1.04468 & -0.10643 & 1.05259 & -0.11079 & 1.05738 \\
\cline { 2 - 8 } & 0.75 & -0.13094 & 1.08247 & -0.14643 & 1.10395 & -0.15396 & 1.11448 \\
\cline { 2 - 8 } & 1 & -0.14325 & 1.09949 & -0.15993 & 1.1227 & -0.1676 & 1.13312 \\
\hline
\end{tabular}




\begin{tabular}{|l|l|l|l|l|l|l|l|}
\hline & 1.25 & -0.15351 & 1.11385 & -0.17033 & 1.13681 & -0.17638 & 1.14504 \\
\hline \multirow{4}{*}{0.25} & 0.25 & -0.10156 & 1.04354 & -0.10988 & 1.05145 & -0.11461 & 1.05628 \\
\cline { 2 - 8 } & 0.75 & -0.13679 & 1.08218 & -0.1533 & 1.10463 & -0.16038 & 1.11491 \\
\cline { 2 - 8 } & 1 & -0.15005 & 1.10003 & -0.16511 & 1.12195 & -0.16952 & 1.12862 \\
\cline { 2 - 8 } & 1.25 & -0.15999 & 1.11433 & -0.17054 & 1.13018 & -0.17171 & 1.13197 \\
\hline \multirow{4}{*}{0.5} & 0.25 & -0.09818 & 1.03781 & -0.10606 & 1.04446 & -0.11049 & 1.04847 \\
\cline { 2 - 8 } & 0.75 & -0.13053 & 1.06914 & -0.14332 & 1.0846 & -0.14745 & 1.08998 \\
\cline { 2 - 8 } & 1 & -0.14108 & 1.08176 & -0.14939 & 1.09256 & -0.15037 & 1.09388 \\
\cline { 2 - 8 } & 1.25 & -0.14726 & 1.08972 & -0.15045 & 1.09399 & -0.15049 & 1.09404 \\
\hline \multirow{4}{*}{1} & 0.25 & -0.08022 & 1.02348 & -0.08561 & 1.02684 & -0.0885 & 1.02875 \\
\cline { 2 - 8 } & 0.75 & -0.09963 & 1.03678 & -0.10388 & 1.04014 & -0.10445 & 1.04061 \\
\cline { 2 - 8 } & 1 & -0.10338 & 1.03973 & -0.10455 & 1.04069 & -0.10457 & 1.0407 \\
\cline { 2 - 8 } & 1.25 & -0.10443 & 1.04059 & -0.10457 & 1.0407 & -0.10457 & 1.0407 \\
\hline \multirow{4}{*}{1.5} & 0.25 & -0.05988 & 1.01262 & -0.06284 & 1.01392 & -0.06432 & 1.01459 \\
\cline { 2 - 8 } & 0.75 & -0.06879 & 1.01673 & -0.06966 & 1.01716 & -0.0697 & 1.01718 \\
\cline { 2 - 8 } & 1 & -0.0696 & 1.01713 & -0.0697 & 1.01718 & -0.0697 & 1.01718 \\
\cline { 2 - 8 } & 1.25 & -0.0697 & 1.01718 & -0.0697 & 1.01718 & -0.0697 & 1.01718 \\
\hline
\end{tabular}

The values in Table 2 indicate a narrow range for the skewness of $\mathrm{X}$, similary, the kurtosis does not vary so much.

\subsection{Order statistics}

In this subsection, we derive the pdf of the $s$ th $(1 \leq s \leq n)$ order statistics $X_{s: n}$, $\mathrm{g}_{s: n}$ say, is defined as

$$
\mathrm{g}_{s: n}(t)=\frac{1}{B(s, n-s+1)} \mathrm{g}(t) G^{s-1}(t)\{1-G(t)\}^{n-s},
$$

where, $B(s, n-s+1)$ is the beta function. Expanding the binomial expansion. We get

$$
\mathrm{g}_{s: n}(t)=\frac{1}{B(s, n-s+1)} \sum_{i=1}^{n-s}(-1)^{i}\left(\begin{array}{c}
n-s \\
i
\end{array}\right)\left(\frac{F(t, \theta)}{F(\zeta, \theta)}\right)^{s+i} \frac{f(t, \theta)}{F(t, \theta)},
$$


where, $f(t, \theta)$ and $F(t, \theta)$ are the pdf and cdfs of the power Lindley distribution. For $s=1$, particularly the pdf of the first order statistics is given by

$$
\mathrm{g}_{1: n}(t)=n \sum_{i=1}^{n-s}(-1)^{i}\left(\begin{array}{c}
n-1 \\
i
\end{array}\right) \frac{F^{i}(t, \theta) f(t, \theta)}{F^{i+1}(\zeta, \theta)}
$$

Substituting, the pdf and cdfs of the power Lindley distribution, we obtained

$$
\mathrm{g}_{1: n}(t)=\frac{\theta^{2} \beta}{1+\theta} \sum_{i=1}^{n-1} \frac{(-1)^{i} x^{\beta-1}\left(1+x^{\beta}\right) e^{-\theta x^{\beta}}\left[1-\left(1+\frac{\theta x^{\beta}}{\theta+1}\right) e^{-\theta x^{\beta}}\right]^{i}}{B(n-1, i+1)\left[1-\left(1+\frac{\theta \zeta^{\beta}}{\theta+1}\right) e^{-\theta \zeta^{\beta}}\right]^{i+1}}
$$

Similarly, the pdf of $X_{n: n}$ is given by

$$
\mathrm{g}_{n: n}(t)=\frac{n \theta^{2} \beta x^{\beta-1}\left(1+x^{\beta}\right) e^{-\theta x^{\beta}}\left[1-\left(1+\frac{\theta x^{\beta}}{\theta+1}\right) e^{-\theta x^{\beta}}\right]^{n-1}}{(1+\theta)\left[1-\left(1+\frac{\theta \zeta^{\beta}}{\theta+1}\right) e^{-\theta \zeta^{\beta}}\right]^{n}}
$$

The mean and variance of the sth order statistics can be obtained by using the formulae used in section 3.1 for UTPL distribution.

\section{Maximum likelihood estimation}

In this section, we describe the procedure to obtain the maximum likelihood estimates (MLE) of the parameters of UTPLD as well as lower truncated power Lindley (LTPLD) and double truncated power Lindley (DTLD) distributions based on the random sample $X=\left\{x_{1}, x_{2}, \ldots, x_{n}\right\}$ of size $n$, so that these distributions can be effectively used to model the real problems depending upon the nature of the data. We fitted these distribution to a set of real data in next section.

\subsection{MLEs for UTPLD}


Let $X$ be an iid (independent and identically distributed) sample of size $n$ from UTPL distribution. The likelihood function based on the observed sample $X$ is given by

$L(\theta, \beta, \zeta \mid X)=\left[\frac{\theta^{2} \beta}{(\theta+1)\left(\exp \left(\theta \zeta^{\beta}\right)-1\right)-\theta \zeta^{\beta}}\right]^{n} \prod_{i=1}^{n}\left(1+x_{i}^{\beta}\right) x_{i}^{\beta-1} e^{-\theta \sum_{i=1}^{n}\left(x_{i}^{\beta}-\zeta^{\beta}\right)}$

$\ln L=$

$$
\begin{array}{r}
2 n \ln \theta+n \ln \beta-n \ln \left[(\theta+1)\left(\exp \left(\theta \zeta^{\beta}\right)-1\right)-\theta \zeta^{\beta}\right]+\sum_{i=1}^{n} \ln \left(1+x_{i}^{\beta}\right) \\
+(\beta-1) \sum_{i=1}^{n} \ln \left(x_{i}\right)-\theta \sum_{i=1}^{n} x_{i}^{\beta}+n \theta \zeta^{\beta}
\end{array}
$$

Note that in the above log-likelihood equation (24), it is not possible to get an estimate of $\zeta$ In terms of observed sample since $\zeta$ is free from $x$. Now, from the order statistics, let $x_{(1)}<x_{(2)}<\cdots<x_{(n)}$ be the order sample corresponding to $x_{1}, x_{2}, \ldots, x_{n}$. Then, the MLE $\hat{\zeta}$ of $\zeta$ can be taken as $\hat{\zeta}=\max \left(x_{1}, x_{2}, \ldots, x_{n}\right)$ i.e. $\hat{\zeta}=x_{(n)}$ largest observation. Once, we get the MLE of $\zeta$. The MLEs $\hat{\theta}, \hat{\beta}$ of $\theta, \beta$ are then the solutions of the following non-linear equations:

$$
\begin{array}{r}
\frac{\partial}{\partial \beta} \ln L=\frac{n}{\beta}-\frac{n\left(\theta \zeta^{\beta} \cdot \ln \zeta-\theta \zeta^{\beta} \mathrm{e}^{\theta \zeta^{\beta}} \cdot \ln \zeta \cdot(\theta+1)\right)}{(\theta+1)\left(\mathrm{e}^{\theta \zeta^{\beta}}-1\right)-\theta \zeta^{\beta}}+\sum_{i=1}^{n} \frac{x_{i}^{\beta} \ln \left(x_{i}\right)}{1+x_{i}^{\beta}}+\sum_{i=1}^{n} \ln \left(x_{i}\right) \\
-\theta \sum_{i=1}^{n} x_{i}^{\beta} \ln \left(x_{i}\right)+n \theta \zeta^{\beta} \ln \zeta=0 \\
\frac{\partial}{\partial \theta} \ln L=\frac{2 n}{\theta}+\frac{n\left[\mathrm{e}^{\theta \zeta^{\beta}}\left(1+\zeta^{\beta}(\theta+1)\right)-\zeta^{\beta}-1\right]}{(\theta+1)\left(\mathrm{e}^{\theta \zeta^{\beta}}-1\right)-\theta \zeta^{\beta}}-\sum_{i=1}^{n} x_{i}^{\beta}+n \zeta^{\beta}=0
\end{array}
$$

In order to solve the above equation, we need to use the iterative procedure like Newton's method.

\subsection{MLEs for LTPLD}

The likelihood function based on $X$ from LTPL distribution is given by 
$L(\theta, \beta, v \mid X)=\left[\frac{\theta^{2} \beta}{\left(1+\theta+\theta v^{\beta}\right)}\right]^{n} \prod_{i=1}^{n}\left(1+x_{i}^{\beta}\right) x_{i}^{\beta-1} e^{-\theta \sum_{i=1}^{n}\left(x_{i}^{\beta}-v^{\beta}\right)}$

The log-likelihood equation is given by

$$
\begin{aligned}
\ln L=2 n \ln \theta+n \ln \beta-n & \ln \left(1+\theta+\theta v^{\beta}\right)+\sum_{i=1}^{n} \ln \left(1+x_{i}^{\beta}\right) \\
& +(\beta-1) \sum_{i=1}^{n} \ln \left(x_{i}\right)-\theta \sum_{i=1}^{n} x_{i}^{\beta}+n \theta v^{\beta}
\end{aligned}
$$

Similarly from the above subsection. The maximum likelihood estimate of $v$ will be $\hat{v}=\min \left(x_{i}\right) ; i=1,2, \ldots, n$ smallest observation. Once, we get the MLE of $v$.

The MLEs $\hat{\theta}, \hat{\beta}$ of $\theta, \beta$ are then the solutions of the following non-linear equations:

$\frac{\partial}{\partial \beta} \ln L=\frac{n}{\beta}+\sum_{i=1}^{n} \frac{x_{i}^{\beta} \ln \left(x_{i}\right)}{1+x_{i}^{\beta}}+\sum_{i=1}^{n} \ln \left(x_{i}\right)-\theta \sum_{i=1}^{n} x_{i}^{\beta} \ln \left(x_{i}\right)+n \theta v^{\beta} \ln v=0$

$\frac{\partial}{\partial \theta} \ln L=\frac{2 n}{\theta}-\frac{n\left(1+v^{\beta}\right)}{\left(1+\theta+\theta v^{\beta}\right)}-\sum_{i=1}^{n} x_{i}^{\beta}+n v^{\beta}=0$

The last equation has a unique solution in $\theta$ given by

$$
\hat{\theta}(\hat{\beta})=\frac{-\left(\sum_{i=1}^{n} \frac{x_{i}^{\beta}}{n}-2 v^{\beta}-1\right)+\sqrt{\left(\sum_{i=1}^{n} \frac{x_{i}^{\beta}}{n}\right)^{2}+2 \sum_{i=1}^{n} \frac{x_{i}^{\beta}}{n}\left(2 v^{\beta}+3\right)-4 v^{\beta}\left(v^{\beta}+1\right)+1}}{2\left(1+v^{\beta}\right)\left(\sum_{i=1}^{n} \frac{x_{i}^{\beta}}{n}-v^{\beta}\right)}
$$

where $\hat{\beta}$ is the solution of the following non-linear equation $G(\beta)=\frac{n}{\beta}+\sum_{i=1}^{n} \frac{x_{i}^{\beta} \ln \left(x_{i}\right)}{1+x_{i}^{\beta}}+\sum_{i=1}^{n} \ln \left(x_{i}\right)-\hat{\theta}(\hat{\beta}) \sum_{i=1}^{n} x_{i}^{\beta} \ln \left(x_{i}\right)+n \hat{\theta}(\hat{\beta}) v^{\beta} \ln v=0$ 


\subsection{MLEs for DTPLD}

The likelihood function under the assumption of the double truncated power Lindley distribution for the random variable $X$, is given by

$L(\theta, \beta, \zeta, v \mid X)=\left[\frac{\theta^{2} \beta}{\phi(\theta, \beta)^{n}}\right]^{n} \prod_{i=1}^{n}\left(1+x_{i}^{\beta}\right) x_{i}^{\beta-1} e^{-\theta \sum_{i=1}^{n}\left(x_{i}^{\beta}\right)}$,

where, $\phi(\theta, \beta)=(1+\theta)\left(e^{-\theta v^{\beta}}-e^{-\theta \zeta^{\beta}}\right)+\theta\left(v^{\beta} e^{-\theta v^{\beta}}-\zeta^{\beta} e^{-\theta \zeta^{\beta}}\right)$.

The corresponding log-likelihood function is given by

$$
\begin{aligned}
\ln L=2 n \ln \theta+n \ln \beta-n \ln (\phi(\theta, \beta)) & +\sum_{i=1}^{n} \ln \left(1+x_{i}^{\beta}\right) \\
& +(\beta-1) \sum_{i=1}^{n} \ln \left(x_{i}\right)-\theta \sum_{i=1}^{n} x_{i}^{\beta}
\end{aligned}
$$

For given MLEs of $v$ and $\zeta$ as $\hat{v}=x_{(1)}$ and $\hat{\zeta}=x_{(n)}$. Respectively, the MLE of $\theta, \beta$ can be obtained by solving the following log-likelihood equations:

$\frac{\partial}{\partial \beta} \ln L=\frac{n}{\beta}+\frac{n \phi^{\prime}(\beta)}{\phi(\theta, \beta)}+\sum_{i=1}^{n} \frac{x_{i}^{\beta} \ln \left(x_{i}\right)}{1+x_{i}^{\beta}}+\sum_{i=1}^{n} \ln \left(x_{i}\right)-\theta \sum_{i=1}^{n} x_{i}^{\beta} \ln \left(x_{i}\right)$

where $\phi^{\prime}(\beta)=\frac{d \phi(\theta, \beta)}{d \beta}$

$\frac{\partial}{\partial \theta} \ln L=\frac{2 n}{\theta}-\frac{n \phi^{\prime}(\theta)}{\phi(\theta, \beta)}-\sum_{i=1}^{n} x_{i}^{\beta}=0$,

where $\phi^{\prime}(\theta)=\frac{d \phi(\theta, \beta)}{d \theta}$

\section{5. real data Analysis}

In this section, we provide applications to a real data set to demonstrate the potentiality of the truncated versions of power Lindley distribution. A set of 
real test data representing the strength data of glass of the aircraft window reported by (1994) is used to evaluate the performance of the truncated power Lindley distribution. It consists of the observations listed in Table 3. Then, the descriptive statistics for the data of the window strengths are given in Table 4.

We fitted the data by exponential, Weibull, Lindley, upper truncated Lindley, power Lindley and lower, upper and double truncated power Lindley distributions. The distribution function of the Weibull model is defined as

$$
F(x)=1-\exp \left(-\theta x^{\beta}\right) \quad x, \theta, \beta>0
$$

Table 3: strength data of glass of the aircraft window

\begin{tabular}{|l|l|l|l|l|l|l|l|l|l|l|}
\hline 18.83 & 26.78 & 36.98 & 20.80 & 27.05 & 37.08 & 21.657 & 27.67 & 37.09 & 23.03 & 25.52 \\
\hline 23.23 & 31.11 & 44.045 & 24.05 & 33.20 & 45.29 & 24.321 & 33.73 & 45.381 & 25.5 & \\
\hline 33.890 & 25.80 & 34.76 & 26.69 & 35.75 & 26.770 & 35.91 & 29.90 & 33.76 & 39.58 & \\
\hline
\end{tabular}

Table 4: Descriptive statistics for the strength data of glass of the aircraft window

\begin{tabular}{|l|c|c|c|c|c|c|l|}
\hline $\mathrm{n}$ & Minimum & Median & Mean & Maximum & $1^{\text {st }} \mathrm{Qu}$ & 3rd Qu & variance \\
\hline 31 & 18.83 & 29.90 & 30.81 & 45.381 & 25.51 & 35.83 & 52.61 \\
\hline
\end{tabular}

We estimate the unknown parameters of the distributions by the maximum likelihood. Table 5 represents the fitting summary of these distributions including the estimates of parameters, log-likelihood, Akaike information criterion (AIC), Corrected Akaike information criterion (AICC), and Bayesian information criterion (BIC) which are calculated by given equations,

$$
\begin{aligned}
& A I C=-2 \log (L)+2 k, \\
& A I C C=A I C+\frac{2 k(k+1)}{(n-k-1)} \\
& B I C=-2 \log (L)+k \log (n)
\end{aligned}
$$


Where $k$ is the number of parameters and $n$ is the sample size.

According to the AIC, AICC and BIC statistics values, the DTPL distribution gives the best fitting to the real data.

Table 5: Maximum likelihood estimates, AIC, AICC, BIC statistics values under considered distributions based on real data

\begin{tabular}{|c|c|c|c|c|c|}
\hline Distribution & Parameter Estimations & $-\log L$ & AIC & AICC & $\mathrm{BIC}$ \\
\hline $\operatorname{Exp}(\theta)$ & $\hat{\theta}=0.03246$ & 137.264 & 276.529 & 276.667 & 277.512 \\
\hline$W(\theta, \beta)$ & $(\hat{\theta}, \hat{\beta})=(0.00017,2.5046)$ & 113.067 & 230.135 & 230.563 & 229.117 \\
\hline$L(\theta)$ & $(\hat{\theta})=0.06299$ & 126.994 & 255.988 & 256.126 & 256.971 \\
\hline $\operatorname{UTL}(\theta, \zeta)$ & $(\hat{\theta}, \hat{\zeta})=(0.00754,45.381)$ & 110.216 & 224.431 & 224.860 & 223.414 \\
\hline$P L(\theta, \beta)$ & $(\hat{\theta}, \hat{\beta})=(0.00023,2.61718)$ & 105.736 & 215.473 & 215.901 & 214.455 \\
\hline $\operatorname{UTPL}(\theta, \beta, \zeta)$ & $\begin{array}{l}(\hat{\theta}, \hat{\beta}, \hat{\zeta}) \\
=(0.000114,2.778283,45.381\end{array}$ & 103.858 & 213.716 & 214.605 & 212.190 \\
\hline $\operatorname{LTPL}(\theta, \beta, v)$ & $\begin{array}{l}(\hat{\theta}, \hat{\beta}, \hat{v}) \\
=(0.000309,2.557831,18.83)\end{array}$ & 102.639 & 211.278 & 212.167 & 209.752 \\
\hline $\operatorname{DTPL}(\theta, \beta, \nu, \zeta$ & $\begin{array}{l}(\hat{\theta}, \hat{\beta}, \hat{v}, \hat{\zeta}) \\
=(0.0038,1.7960,18.83,45.38)\end{array}$ & 100.867 & 209.732 & 211.270 & 207.697 \\
\hline
\end{tabular}

(AIC\& BIC etc.) of the different truncated forms of the power Lindley distribution can be diagrammatically shown as

Worst power Lindley $\rightarrow$ UTP Lindley $\rightarrow$ LTP Lindley $\rightarrow$ DTP Lindley best

\section{Conclusion}

In this paper, we propose a new model called the Upper truncated power Lindley distribution. We investigate several structural properties of the new distribution, expressions for the moments, quantile function, order statistics, 
survival function, hazard function and the moment generating function. The estimation of parameters is approached by the method of maximum likelihood. the data of the window strengths is modeled by the lower, upper and double truncated power Lindley distributions, exponential, Lindley, upper truncated Lindley, power Lindley and Weibull distributions to evaluate the performance of the truncated versions of power Lindley distribution. Fitting performance of these distributions is compared according to AIC, AICC and BIC statistics values and clearly the data of the window strengths is best modeled by DTPL distribution. Finally, it is concluded that the truncated distributions can be quit effectively used to model the real problems and so we can recommend the use of the truncated power Lindley distributions in various fields including engineering, medical, finance and demography where such type of truncated data are commonly encountered. 


\section{References}

Ahmed, S.E., Castro-Kuriss, C., Leiva, V., Sanhueza, A. (2010). A truncated version of the Birnbaum-Saunders distribution with an application in financial risk, Pakistan Journal of Statistics, 26(1), 293-311.

Asgharzadeh, A., Bakouch, S.H., Nadarajah, S., Esmaeili. L.A. (2014). New family of compound lifetime distributions. Kybernetika.; 50 (1): 142-169.

Ashour, K, S., Eltehiwy, A, M. (2015). Exponentiated Power Lindley Distribution. Journal of Advanced Research; 6, 895-905.

Bakouch, H.S., Bander, M.A., Al-Shaomrani, A.A. Marchi, V.A.A. and Louzada, F. (2012). An extended Lindley distribution, Journal of The Korean Statistical Society, 41, 75-85.

Corless, R.M., Gonnet, G.H., Hare, D.E., Jeffrey, D. J., Knuth, D.E. (1996). On the Lambert W function. Advances in Computational mathematics, 5(1), 329-359.

Fuller-Jr, E. Frieman, S., Quinn, J., Quinn, G., Carter, W. (1994). Fracture mechanics approach to the design of glass aircraft windows: A case study, SPIE Proc 2286: 419-430.

Ghitany, M.E., Atieh, B., Nadarajah, S. (2008). Lindley distribution and its application. Mathematics and computers in simulation, 78(4), 493-506.

Ghitany, M., Al-Mutairi, D., Balakrishnan, N., Al-Enezi, L. (2013). Power Lindley distribution and associated inference. Computational Statistics and Data Analysis, $64,20-33$. 
Ghitany, M.E., Al-qallaf, F, Al-Mutairi D.K., Hussain H. A. (2011). A two parameter weighted Lindley distribution and its applications to survival data. Mathematics and computers in simulation.; 81(6):1190-1201.

Jodrá, J. (2010). Computer generation of random variables with Lindley or Poisson-Lindley distribution via the Lambert $\mathrm{W}$ function. Mathematics and computers in simulation; 81:851-859.

Lindley, D.V. (1958). Fiducial distributions and Bayes Theorem. Journal of the Royal Statistical Society. Series B (Methodological), 102-107.

Merovci, F, Elbatal, I. (2014). Transmuted Lindley- geometric and its application. Journal of Statistics and application and application. 3 (1): 77-91.

Nadarajah, S., Bakouch, H.S., Tahmasbi, R.A. (2011) Generalized Lindley distribution. Sankhya B ; 73: 331-359.

Oluyede, B, Yang, T. A. (2015). New class of generalized Lindley distributions with applications. Journal of statistical computation and simulation. 85, 10.

Singh, S. K., Singh, U., Sharma, V. K., (2014). The Truncated Lindley Distribution: Inference and Application, Journal of Statistics Applications \& Probability, 3(2), 219-228.

Zhang T. and Xie M., (2011). On the upper truncated Weibull distribution and its reliability implications, Reliability Engineering and System Safety, 96, 94-200. 